\title{
Empirically derived guidelines for interpreting the effectiveness of exercise therapy for tendinopathies: A protocol.
}

Swinton, P.A. ${ }^{*}$ Shim, J. ${ }^{1}$ Pavlova, A.V. ${ }^{1}$ Moss, R.A. ${ }^{1}$ Maclean, C. ${ }^{2}$ Brandie, D. ${ }^{3}$ Mitchell, L. ${ }^{4}$ Greig, L. ${ }^{1}$

Parkinson, E. ${ }^{1}$ Morrissey, D. ${ }^{5}$ Alexander, L. ${ }^{1}$ Cooper, K. ${ }^{1}$

DOI: $10.31236 /$ osf.io/y7sk6

SportR $\chi$ iv hosted preprint version 1

Last updated

PREPRINT - NOT PEER REVIEWED

Institutions

${ }^{1}$ School of Health Sciences, Robert Gordon University, Aberdeen, UK

${ }^{2}$ Library Services, Robert Gordon University, Aberdeen, UK

${ }^{3}$ Sport Scotland, Inverness, UK

${ }^{4}$ NHS Grampian, Aberdeen, UK

${ }^{5}$ Centre for Sports \& Exercise Medicine, Barts \& the London School of Medicine \& Dentistry, London, UK

* Corresponding Author

Dr. Paul Swinton

School of Health Sciences, Robert Gordon University

Garthdee Road

Aberdeen, UK,

AB10 7QG

p.swinton@rgu.ac.uk, +44 (0) 12242623361

Funding

This project is funded by the National Institute for Health Research (NIHR) [Health Technology Assessment (HTA) 129388 Exercise therapy for the treatment of tendinopathies]. The views expressed are those of the authors and not necessarily those of the NIHR or the Department of Health and Social Care.

\section{Conflicts of interest}

The authors declare no conflict of interest. 


\subsection{Introduction}

Tendinopathy is a common musculoskeletal condition associated with degenerative changes within a tendon affecting both athletic and non-athletic populations. ${ }^{1}$ The condition is characterized by a combination of pain, ${ }^{1}$ and impaired movement ${ }^{2}$ and function ${ }^{3}$, requiring extended periods for recovery. ${ }^{2,4-5}$ Tendinopathy can affect any muscle-tendon unit in the body, ${ }^{6}$ however, it is most frequently reported in the Achilles, patellar, lateral elbow, rotator cuff, and hip tendons. ${ }^{6}$ Surveys of prevalence of lower extremity tendinopathy in the general population have reported rates of 11.8 and 10.5 per 1000 person-years, ${ }^{7}$ whilst prevalence for upper limb tendinopathies have been estimated between $1.3 \%$ to $21.0 \% .^{8-10}$ Tendinopathies can affect children, adolescents, and adults of all ages, and many tendinopathies have a chronic or recurrent course ${ }^{6}$ Costs to the individual, the health service and economy (due to absenteeism and loss of productivity) are substantial such that identifying effective interventions is a priority. Musculoskeletal conditions including tendinopathies also have a substantive influence on primary and secondary healthcare use. ${ }^{11}$ By identifying effective interventions across a range of tendinopathies, General Practitioners and other first-contact practitioners (e.g. physiotherapists) can be confident in delivering effective evidence-based practice. With an ageing population, and increasing pressure and demands on healthcare services, the need for clear guidance for evidence-based practice has never been more important.

Exercise therapy is the mainstay of conservative management of tendinopathy and has focused largely on resistance training, and in many instances eccentric strengthening techniques, to date. ${ }^{12}$ The objective with exercise therapy is to encourage load tolerance that leads to structural adaptation at the musculotendinous unit and restores function. ${ }^{13-14}$ Isometric, isotonic, and heavy slow resistance training have also been recommended for some tendinopathies (e.g. patellar) with suggested efficacy. ${ }^{15}$ In the early phase of rehabilitation, range of movement and flexibility exercises are often initiated and incorporated into strengthening regimes to facilitate improvements in mobility. ${ }^{12}$ Included exercises range from static stretches to ballistic actions and variations of contract-relax stretching adapted from the proprioceptive neuromuscular facilitation literature. ${ }^{12}$ Effective exercise therapy may also require targeting a range of contributing factors, which not only include muscle weakness and decreased flexibility, but also corticospinal and neuromuscular adaptations resulting from persistent pain. ${ }^{16}$ As such, movement retraining or motor control-based exercise interventions have been used to retrain normal patterns of muscle recruitment in the rehabilitation of shoulder-related tendinopathies including impingement, with supportive evidence provided in trials and systematic reviews. ${ }^{16-19}$ Similarly, balance and core stabilisation exercises have been recommended for patients presenting with lumbo-pelvic instability in conjunction with patellar and Achilles tendinopathy. ${ }^{20}$ Whilst various exercise therapies have been proposed for the treatment of tendinopathy and the overarching aims of reducing pain and disability, and improving function, recommendations are frequently equivocal with no consensus on treatment guidelines for major tendinopathies.

The large range of outcomes reported across research investigating the treatment of tendinopathies has created barriers to the evaluation and synthesis of effectiveness data. Attempts have been made to develop core domains to facilitate synthesis of outcomes measuring similar constructs. ${ }^{21}$ Synthesis has been most effective when conducting meta-analyses, where previous studies have generally quantified effect sizes through calculation of standardised mean differences. The most common approach used to interpret the magnitude of effect sizes and Swinton, Shim, Pavlova, Moss, Maclean, Greig, Parkinson, Morrissey, Alexander, Cooper (2021) 
therein the clinical effectiveness of a therapy has been the use of the Cohen's standard benchmarks (small $=0.2$, medium $=0.5$ and large $=0.8)$ irrespective of the tendinopathy type, therapy, or population. ${ }^{22-30}$ Despite Cohen's

31 recommendations that his general benchmarks should only be used when more relevant context specific information is unavailable, use of these standard benchmarks is ubiquitous throughout behavioural, social and health sciences. However, recent attempts have been made across a range of disciplines to use empirically derived effect size distributions to generate context specific benchmarks. ${ }^{32-38}$ Results have generally demonstrated substantive differences between Cohen's benchmarks and those derived empirically, with examples of both under- and over-estimation. In addition, research has shown that even within a single discipline, substantive differences in the distribution of effect sizes can exist across sub-domains. ${ }^{32}$ Given the range of tendinopathies and outcome domains commonly investigated, there is potential that the distribution and subsequent appropriate interpretation of therapy effects will be diverse and benefit from the generation of context specific benchmarks. Therefore, the purpose of this study is to perform a large synthesis of the available research investigating exercise therapy for tendinopathies, creating empirically derived thresholds to benchmark interventions and explore potential differences across tendinopathy types and outcome domains.

\subsection{Inclusion criteria}

This review is part of a project funded by the National Institute for Health Research (NIHR); Health Technology Assessment (HTA) 129388 Exercise therapy for the treatment of tendinopathies. The inclusion criteria are influenced by the project aims, the results of our initial scoping review mapping the exercise and tendinopathy literature 33 as well as stakeholder workshops.

\section{Participants}

This review will include people of any age or gender with a diagnosis of rotator cuff, lateral elbow, patellar or Achilles tendinopathy of any severity or duration. Studies that include participants with tendinopathy in the absence of full thickness or large tears, will be included. Groups where the tear size cannot be determined will be excluded as these require different management approaches. We will accept trial authors' diagnoses where a clearly verifiable group of clinical features is reported including: pathognomonic location of pain; a symptom altering response to applied load and/or stretch, with there being a specific test for most tendinopathies; strategies to rule out differential diagnoses; ultrasound or magnetic resonance imaging confirmation of structural change. Studies with mixed groups will have data included where there is clear reporting of the tendinopathic group, or they make up $>90 \%$ of the investigated cohort.

\section{Intervention}

The intervention being assessed is exercise therapy and will comprise five treatment classes: 1) resistance; 2) plyometric; 3) vibration; 4) flexibility and 5) proprioception (see appendix I for definitions). Interventions combining exercise with other active therapies (e.g. laser, shockwave, manual therapy or injection) will not be included. Exercise therapy may be delivered in a range of settings (e.g. primary care, secondary care, community, people's homes) by a range of health or exercise professionals (e.g. physiotherapists, strength \& conditioning 
DOI | SportsRxiv Preprint version 1

coaches, personal trainers) or support workers, and may be supervised or unsupervised (i.e. self-management). No restrictions will be placed on these factors for inclusion.

To be included in the review, studies are required to report sufficient information regarding the exercise intervention to enable appropriate identification of treatment duration, treatment class and exercise dose. In clinical settings it has been recommended that exercise dose is determined by duration, frequency, and intensity; where duration reflects the amount of time accrued in a single exercise session, frequency captures the number of exercise sessions over periods such as a week, and exercise intensity is defined either in absolute terms (such as the metabolic cost of an exercise session), or in relative terms (such as the performance of a given activity as a function of some percentage of measurable maximum capacity. To be included in the review, studies are required to provide sufficient information to describe at least two of the three parameters describing exercise dose. Where sufficient information is not presented in the main text of a study, a search will be made of the publishers' website to check for supplementary files that may include relevant information.

Comparator

Both non-controlled (exercise therapy only) and controlled (comparator adjusted) effects will be calculated. The comparator used for controlled effects will include placebo interventions and no treatment.

Outcomes

Based on the results of our initial scoping review and subsequent stake holder workshops we will include outcomes that assess five domains: 1) Disability; 2) Physical function capacity; 3) Pain on loading/activity; 4) Pain over a specified time; and 5) Pain without further specification. Definitions for each domain and example tools are presented in appendix II.

Types of studies

We will include randomized controlled trials and non-randomized controlled trials where at least one intervention arm comprises an exercise therapy that can be categorized according to the treatment classes outlined.

Context

The context will include primary care, secondary care or community locations in any developed nation (defined as the top 62 countries in the Human Development Index at the time of protocol development ${ }^{40}$ for the findings to be relevant to the UK context.

\subsection{Exclusion criteria}

We will exclude self-described pilot studies and non-intervention studies where the purpose of the research is to investigate the acute effects of exercise on physiological or biomechanical variables such as pain, collagen turnover or mechanical properties of tendons. 


\subsection{Methods}

Search strategy

The search strategy used for this study was part of a larger search conducted to scope the entire tendinopathy and exercise therapy research base. The search comprised three steps; Firstly, a limited search of MEDLINE and CINAHL using initial keywords (MH tendinopathy OR TX tendin* OR TX tendon*) AND (MH exercise OR TX exercis*) was conducted with analysis of the text words in the titles/abstracts and those used to describe articles to develop a full search strategy. Secondly, the full search strategy was adapted to each database and applied systematically to: MEDLINE, CINAHL, AMED, EMBase, SPORTDiscus, Cochrane library (Controlled trials, Systematic reviews), JBI Evidence Synthesis, PEDRo, and Epistemonikos (a full search strategy for MEDLINE is presented in appendix III). The following trial registries were also searched: ClinicalTrials.gov, ISRCTN Registry, The Research Registry, EU-CTR (European Union Clinical trials Registry), ANZCTR (Australia and New Zealand Clinical trials Registry). Finally, the third step involved conducting a search of cited and citing articles using Scopus and hand-searching a total of 130 systematic reviews that were identified to include information relevant to exercise therapy and tendinopathy. No limit was placed on language, with research studies published in languages other than English translated via Google Translate or via international collaborations of the review team members. Searches were initiated from 1998 as (i) the heavy load eccentric calf-training protocol for Achilles tendinosis by Alfredsson et al ${ }^{41}$ was published in 1998 and may be considered seminal work in the field of tendinopathy, and (ii) there has been a proliferation of research on exercise interventions for tendinopathies post 1998.

Study selection

Proquest ${ }^{\circledR}$ Refworks will be used to manage references and remove duplicates, before importing to Covidence (Melbourne, Australia) to facilitate screening and initiate a second deduplication process. Two levels of screening will be conducted. First, all titles/abstracts will be reviewed, independently, by two members of the research team. Conflicts will be resolved by discussion or by input from a third reviewer. Second, full-text copies of all studies included at title/abstract screening stage will be retrieved and these will also be screened independently by two members of the research team with conflicts resolved in the same way.

\section{Data extraction}

Data will be extracted independently by 8 members of the review team (PS/KC/LA/RM/LG/EP/JS/AP) into pre-piloted excel sheets. Data will be independently coded as described in the accompanying extraction codebook (appendix IV). To quantify reliability, 10\% of studies will be selected at random and extraction completed in duplicate. Reliability will be quantified using Cohens $\mathrm{K}$ statistic ${ }^{42}$ for categorical variables and percentage agreement for continuous variables.

Statistical analysis

To synthesise effects across different measurement outcomes within each domain, standardised mean difference $\left(\mathrm{SMD}_{\text {pre }}\right)$ effect sizes will be calculated by dividing the relevant mean difference by the pre-intervention standard Swinton, Shim, Pavlova, Moss, Maclean, Greig, Parkinson, Morrissey, Alexander, Cooper (2021) 
deviation. As standard, non-controlled effect sizes will be calculated for the exercise therapy group by subtracting baseline values from measurements made at subsequent time-points. Where placebo interventions or no treatment arms are included, the mean difference in the comparator will be subtracted from the mean difference in the exercise therapy. Values will then be standardised by dividing by the pooled baseline standard deviation. Where sufficient data is presented for a single measurement tool, non-standardised effect sizes will also be included to facilitate clinical interpretations. Where outcomes are assessed at multiple time-points following baseline measurement, all possible $\mathrm{SMD}_{\text {pre }}$ values will be calculated. Where required, $\mathrm{SMD}_{\text {pre }}$ values will be reflected by multiplying by -1 to ensure that positive values represent a positive clinical effect. Where baseline standard deviation values are not presented these will be estimated using statistical information presented 43 (e.g. confidence intervals, standard errors, $\mathrm{t}$ values, $\mathrm{P}$ values, $\mathrm{F}$ values) or will be imputed through simple linear regression of the log-transformed standard deviations and means from studies included in the same analysis. ${ }^{44}$

The $10^{\text {th }}$ to $90^{\text {th }}$ percentiles will be presented for each effect size distribution in 5-unit increments. In accordance with the most common procedures used in previous studies generating context specific benchmarks, the $25^{\text {th }}$, $50^{\text {th }}$ and $75^{\text {th }}$ percentile values will be used to identify small, medium, and large effect sizes, respectively..$_{4,35,38,45}$ Bayesian three-level hierarchical models with weakly-informative Student-t and half-t priors will be used to

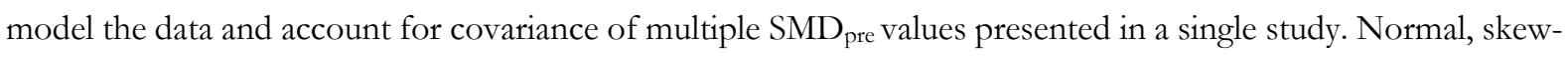
normal and t-distribution models will be assessed for fit, and posterior samples used to report $25^{\text {th }}, 50^{\text {th }}$ and $75^{\text {th }}$ percentile values with credible intervals. Analyses will be performed using the $\mathrm{R}$ wrapper package brms interfaced with Stan to perform Markov chain Monte Carlo sampling. ${ }^{46}$ 


\section{References}

1. Hopkins C, Fu SC, Chua E, Hu X, Rolf C, Mattila VM, et al. Critical review on the socio-economic impact of tendinopathy. Asia Pac J Sports Med Arthrosc Rehabil Technol. 2016; 4: 9-20.

2. Lewis J. Rotator cuff related shoulder pain: Assessment, management and uncertainties. Man Ther. 2016; 23:57-68.

3. Alizadehkhaiyat O, Fisher AC, Kemp GJ. Pain, functional disability and psychologic status in tennis elbow. Clin J Pain. 2007; 23(6): 482-489.

4. Seil R, Litzenburger H, Kohn D. Arthroscopic treatment of chronically painful calcifying tendinitis of the supraspinatus tendon. Arthroscopy. 2006; 22(5): 521-527.

5. Comin J, Malliaras P, Baquie P, Barbour T, Connell D. Return to competitive play after hamstring injuries involving disruption of the central tendon. Am J Sports Med. 2013; 41(1): 111-115.

6. Fu FH, Wang JH-C, Rothrauff BB. BMJ Best Practice Tendinopathy [Internet]. London: BMJ; 2019 [cited 2019 Jul 20]. Available from:

https://bestpractice.bmj.com/topics/en-gb/582

7. Albers IS, Zwerver J, Diercks RL, Dekker JH, Van den Akker-Scheek I. Incidence and prevalence of lower extremity tendinopathy in a Dutch general practice population: a cross sectional study. BMC Musculoskelet Disord. 2016; 17:16.

8. Littlewood C, May S, Walters S. Epidemiology of Rotator Cuff Tendinopathy: A Systematic Review. Shoulder \& Elbow. 2013; 5(4): 256-265.

9. Shiri R, Viikari-Juntura E, Varonen H, Heliövaara M. Prevalence and determinants of lateral and medial epicondylitis: A population study. Am J Epidemiol. 2006; 164(11): 1065-1074

10. Vos T. Years lived with disability (ylds) for 1160 sequelae of 289 diseases and injuries 1990-2010: a systematic analysis for the global burden of disease study 2010. Lancet. 2012; 380(9859):2163-2196.

11. Kinge JM, Knudsen AK, Skirbekk V, Vollset SE. Musculoskeletal disorders in Norway: prevalence of chronicity and use of primary and specialist health care services. BMC Musculoskelet Disord. 2015;16(1):75.

12. Abat F, Alfredson H, Cucciarini M, Madry H, Marmott A, Mouton C, et al. Current trends in tendinopathy: consensus of the ESSKA basic science committee. Part I: biology, biomechanics, anatomy and an exercise-based approach. J Exp Orthop. 2017; 4:18.

13. Alfredson H. The chronic painful Achilles and patellar tendon: Research on basic biology and treatment. Scand J Med Sci Sports. 2005; 15(4): 252-259.

14. O'Neill S, Watson P, Barry S. Why are eccentric exercises effective for Achilles tendinopathy? Int J Sports Phys Ther. 2015; 10(4): 552-62.
15. Lim HY, Wong SH. Effects of isometric, eccentric, or heavy slow resistance exercises on pain and function in individuals with patellar tendinopathy: A systematic review. Physiother Res Int. 2018 Oct;23(4):e1721.

16. Rio E, et al Tendon neuroplastic training: Changing the way we think about tendon rehabilitation: A narrative review Br J Sports Med. 2016; 50(4): 209-215.

17. Worsley et al. Motor control retraining exercises for shoulder impingement: effects on function, muscle activation, and biomechanics in young adults. J Shoulder Elbow Surg. 2013; 22(4): e11-19.

18. Ager et al. The effectiveness of an upper extremity neuromuscular training program on the shoulder function of military members with a rotator cuff tendinopathy: A Pilot Randomized Controlled Trial. Mil Med. 2019; 184(56): e385-e393.

19. Lewis J, McCreesh K, Roy JS, Ginn K. Rotator Cuff Tendinopathy: Navigating the Diagnosis-Management Conundrum. J Orthop Sports Phys Ther. 2015 Nov;45(11):923-37.

20. Dimitrios S. Exercise for tendinopathy. World J Methodol. 2015; 5(2): 51-54.

21. Vicenzio et al. ICON 2019-International Scientific Tendinopathy Symposium Consensus: There are nine core health-related domains for tendinopathy (CORE DOMAINS): Delphi study of healthcare professionals and patients. Br J Sports Med. 2020; 54: 444-451.

22. Shire et al. Specific or general exercise strategy for subacromial impingement syndrome-does it matter? A systematic literature review and meta analysis. BMC Musculoskelet Disord. 2017; 18(1): 158.

23. Mendoça et al. How strong is the evidence that conservative treatment reduces pain and improves function in individuals with patellar tendinopathy? A systematic review of randomised controlled trials including GRADE recommendations. Br J Sports Med. 2020; 54(2). 87-93.

24. Saltychev M, Äärimaa V, Virolainen P, Laimi K. Conservative treatment or surgery for shoulder impingement: systematic review and meta-analysis. Disabil Rehabil. 2015; 37(1): 1-8

25. Bisset L, Paungmali A, Vicenzino B, Beller E, Herbert R. A systematic review and meta-analysis of clinical trials on physical interventions for lateral epicondylalgia. $\mathrm{Br} \mathrm{J}$ Sports Med. 2005; 39(7): 411-422.

26. Hanratty et al. The effectiveness of physiotherapy exercises in subacromial impingement syndrome: A systematic review and meta-analysis. Semin Arthritis Rheum. 2012; 42(3): 297-316.

27. Young JL, Rhon DI, Cleland JA, Snodgrass SJ. The influence of exercise dosing on outcomes in patients with knee disorders: A systematic review. J Orthop Sports Phys Ther. 2018; 48(3): 146-161.

28. Barr S, Cerisola FL, Blanchard V. Effectiveness of corticosteroid injections compared with physiotherapeutic 
interventions for lateral epicondylitis: A systematic review. Physiotherapy. 2009; 95(4): 251-265.

29. Olaussen M, Homedal O, Lindbaek M, Brage S, Solvang $\mathrm{H}$. Treating lateral epicondylitis with corticosteroid injections or non-electrotherapeutical physiotherapy: A systematic review. BMJ Open. 2013; 3(10): e003564.

30. Chen Z, Baker NA. Effectiveness of eccentric strengthening in the treatment of lateral elbow tendinopathy: A systematic review with meta-analysis. J Hand Ther. 2021; 34(1): 18-28.

31. Cohen, J. Statistical power analysis for the behavioral sciences (2nd ed). 1988. Hillsdale, NJ: Erlbaum.

32. Schäfer T, Schwarz MA. The meaningfulness of effect sizes in psychological research: Differences between subdisciplines and the impact of potential biases. Front Psychol. 2019; 10: 813.

33. Bosco FA, Aguinis H, Singh K, Field JG, Pierce CA. Correlational effect size benchmarks. J Appl Psychol. 2015; 100(2): 431-449.

34. Brydges CR. Effect size guidelines, sample size calculations, and statistical power in gerontology. Innov Aging. 2019; 3(4): igz036.

35. Gignac GE, Szodorai ET. Effect size guidelines for individual differences researchers. Pers Individ Differ. 2016; 102: 74-78.

36. Kraft MA. Interpreting effect sizes of education interventions. Educ Res. 2020; 49(4): 241-253.

37. Paterson TA, Harms PD, Steel P, Credé M. An assessment of the magnitude of effect sizes: Evidence from 30 years of meta-analysis in management. J Leadersh Organ Stud. 2015; 23(1): 1-17.
38. Lovakov A, Agadullina ER. Empirically derived guidelines for effect size interpretation in social psychology. Eur J Soc Psychol. 2021; In Press.

39. Alexander et al. Exercise therapy for the treatment of tendinopathies: A scoping review protocol. JBI Evid Synth. 2021; doi: 10.11124/JBIES-20-00175.

40. United Nations Development Programme-Human Development Reports. Human development index: Table 1 human development index and its components 2020; Retrieved from: http://hdrundporg/en/composite/HDI.

41. Alfredson H, Pietilä T, Jonsson P, Lorentzon R. Heavyload eccentric calf muscle training for the treatment of chronic Achilles tendinosis. Am J Sports Med. 1998; 26(3): 360-366.

42. Cohen J. Weighted kappa: nominal scale agreement with provision for scaled disagreement or partial credit. Psychol Bull. 1968; 70: 213-220.

43. Cochrane handbook 7.7.3. https://handbook-51.cochrane.org/chapter_7/7_7_3_data_extraction_for_con tinuous_outcomes.htm.

44. Marinho VCC, Higgins JPT, Logan S, Sheiham A. Fluoride toothpaste for preventing dental caries in children and adolescents. Cochrane Database of Systematic Reviews. 2003; 1:Art No: CD00227850.

45. Quintana DS. Statistical considerations for reporting and planning heart rate variability case-control studies. Psychophysiology. 2017; 5(3): 344-349.

46. Bürkner PC. brms: An R Package for Bayesian Multilevel Models Using Stan. J Stat Softw. 2017; 80(1): 28. 
Appendix I: Definitions use to define exercise treatment classes.

\begin{tabular}{|c|c|}
\hline Treatment Class & Definition \\
\hline Resistance & $\begin{array}{l}\text { Exercise designed primarily to increase strength of muscles by causing them to produce } \\
\text { substantive force against an applied resistance which can take several forms including } \\
\text { the mass of the body or its segments, isoinertial resistance, elastic resistance, or strength } \\
\text { training equipment such as isokinetic devices. In tendinopathy, the stimulus may also be } \\
\text { intended to provoke tendon remodelling, reduce pain and improve function. }\end{array}$ \\
\hline Plyometric & Exercise where a resistance is overcome by a muscle rapidly stretching then shortening \\
\hline Vibration & $\begin{array}{l}\text { Exercise where body segments are held stationary or actively displaced as per definitions } \\
\text { for other treatment classes whilst applying a rapid oscillating resistance }\end{array}$ \\
\hline Flexibility & $\begin{array}{l}\text { Exercise designed to increase joint range of motion and extensibility of muscles and/or } \\
\text { associated tissues. Also referred to as range-of-motion exercises or stretching. }\end{array}$ \\
\hline Proprioception & $\begin{array}{l}\text { Exercise designed to enhance the sensation of the joint relative to body position and } \\
\text { movement, sense of force, and to encourage muscular stabilisation of the joint in the } \\
\text { absence of external stabilising devices e.g. ankle brace. }\end{array}$ \\
\hline
\end{tabular}




\section{Appendix II: Outcome domains and example outcomes included in review.}

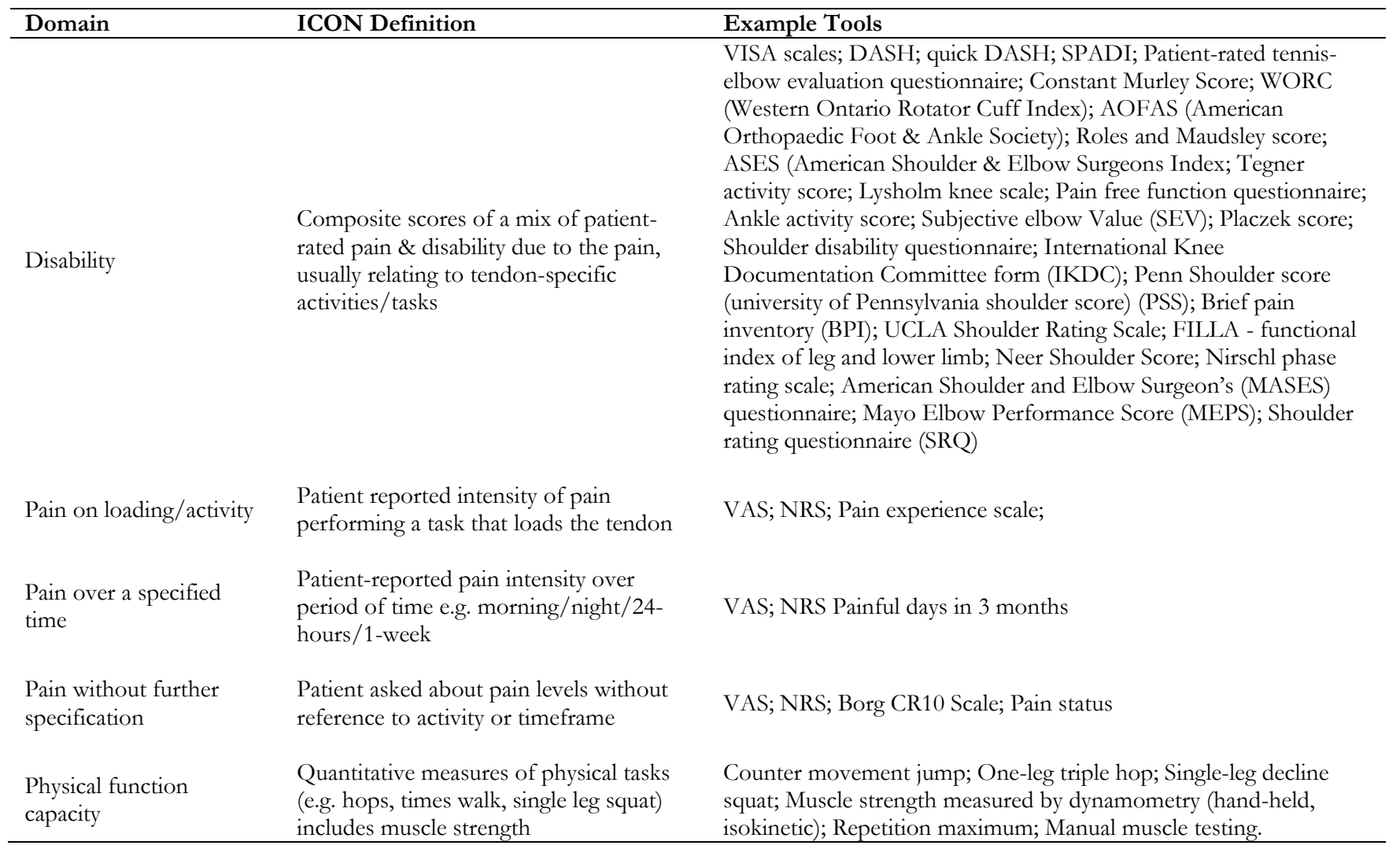


DOI | SportsR $x$ iv Preprint version 1

\section{Appendix III: Search strategy}

MEDLINE (EBSCoHost)

Search conducted on 27 April, 2020

\begin{tabular}{|l|l|l|}
\hline Search & Query & $\begin{array}{l}\text { Records } \\
\text { retrieved }\end{array}$ \\
\hline$\# 1$ & $\begin{array}{l}\text { MH exercise OR AB exercis* OR MH “isometric contraction” OR MH rehabilitation OR TX } \\
\text { eccentric OR TX concentric OR TX “heavy slow resistance” OR TX isokinetic }\end{array}$ & 362,722 \\
\hline$\# 2$ & $\begin{array}{l}\text { MH tendinopathy OR MH “shoulder injuries" OR MH tendons OR MH “tendon injuries” OR } \\
\text { TX tendin* OR TX tendon* OR MH bursitis OR AB bursitis OR MH “posterior tibial tendon } \\
\text { dysfunction" OR MH “shoulder impingement syndrome” OR AB “greater trochanteric pain } \\
\text { syndrome" }\end{array}$ & 96,490 \\
\hline$\# 3$ & $\# 1$ AND \#2 & 4,363 \\
\hline Limited to 1998 to present & \\
\hline
\end{tabular}




\section{Appendix IV: Extraction codebook}

\begin{tabular}{|c|c|c|c|}
\hline \multicolumn{2}{|c|}{ Column } & Heading & Description \\
\hline \multirow{23}{*}{ 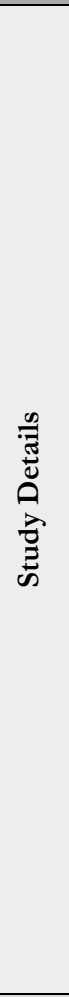 } & A & Initials Reviewer & Identification of individual extracting information \\
\hline & $\mathrm{B}$ & Covidence Identifier & Reference number for Covidence \\
\hline & $\mathrm{C}$ & Author & First author surname et al., \\
\hline & $\mathrm{D}$ & Year & Year of publication \\
\hline & $\mathrm{E}$ & Title & Study title \\
\hline & $\mathrm{F}$ & Country & Country where study was conducted \\
\hline & G & Journal & Journal name \\
\hline & $\mathrm{H}$ & Aims/Purpose & Study aims/purpose \\
\hline & I & Tendinopathy type & $1=$ Achilles; $2=$ Lateral elbow (tennis); $3=$ Patellar; $4=$ Rotator cuff (SI) \\
\hline & $\mathrm{J}$ & Study Design & RCT $=1 ;$ Quasi-experimental $=2$ \\
\hline & $\mathrm{K}$ & Age Mean & Mean age of study sample as a whole \\
\hline & $\mathrm{L}$ & Age SD & Standard deviation age of study sample as a whole \\
\hline & $\mathrm{M}$ & Baseline Total N & Total sample across all interventions measured at baseline \\
\hline & $\mathrm{N}$ & $\begin{array}{l}\text { Training Status } \\
\text { Description }\end{array}$ & Brief description of training status of study sample as a whole \\
\hline & $\mathrm{O}$ & Training Status Code & $1=$ Performance; 2 = Sporting; $3=$ Other \\
\hline & $\mathrm{P}$ & Sex & Percentage female of study sample as a whole \\
\hline & Q & BMI Mean & Mean BMI of study sample as a whole \\
\hline & $\mathrm{R}$ & BMI SD & Standard deviation of BMI of study sample as a whole \\
\hline & $\mathrm{S}$ & Symptom Severity Mean & Mean severity measure at baseline of study sample as a whole \\
\hline & $\mathrm{T}$ & Symptom Severity SD & Standard deviation of severity measure at baseline of study sample as a whole \\
\hline & $\mathrm{U}$ & $\begin{array}{l}\text { Symptom Duration } \\
\text { Mean (Months) }\end{array}$ & Mean symptom duration reported in months \\
\hline & $\mathrm{V}$ & $\begin{array}{l}\text { Symptom Duration SD } \\
\text { (Months) }\end{array}$ & Standard deviation symptom duration reported in months \\
\hline & W & Population Comments & $\begin{array}{l}\text { Any additional information relevant to the participants investigated including } \\
\text { diagnostic criteria }\end{array}$ \\
\hline \multirow{5}{*}{ 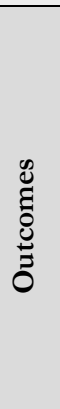 } & $\mathrm{X}$ & Outcome Category & $\begin{array}{c}1=\text { Disability; } 2=\text { Pain on loading/activity; } 3=\text { Pain over a specified time; } 4= \\
\text { Pain without further specification; } 5=\text { Physical function capacity }\end{array}$ \\
\hline & & Outcome Tool & Description of outcome tool \\
\hline & $\mathrm{Y}$ & & \\
\hline & $\mathrm{Z}$ & Reflection & $\begin{array}{c}1 \text { = Increase in outcome indicates positive treatment; }-1=\text { Decrease in outcome } \\
\text { indicates positive treatment }\end{array}$ \\
\hline & $\mathrm{AA}$ & $\begin{array}{l}\text { Measurement Time } \\
\text { (Weeks) }\end{array}$ & Time of measurement in weeks \\
\hline \multirow{12}{*}{ 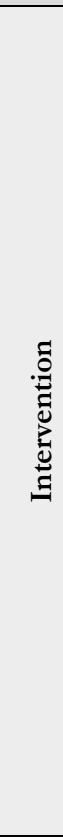 } & $\mathrm{AB}$ & $\begin{array}{l}\text { Dominant Treatment } \\
\text { Class }\end{array}$ & $\begin{array}{c}\text { Only one dominant theme to be selected } \\
1=\text { Resistance; } 2=\text { Plyometric; } 3=\text { Vibration; } 4=\text { Flexibility; } 5=\text { Movement } \\
\text { pattern retraining }\end{array}$ \\
\hline & $\mathrm{AC}$ & Total Treatment class & $\begin{array}{c}\text { Multiple themes to be selected as required } \\
1=\text { Resistance; } 2=\text { Plyometric; } 3=\text { Vibration; } 4=\text { Flexibility; } 5=\text { Movement } \\
\text { pattern retraining }\end{array}$ \\
\hline & $\mathrm{AD}$ & Intervention $\mathrm{N}$ & Intervention sample size at specified time \\
\hline & $\mathrm{AE}$ & $\begin{array}{l}\text { Intervention Total } \\
\text { Duration }\end{array}$ & Total duration of exercise intervention in weeks \\
\hline & $\mathrm{AF}$ & $\begin{array}{l}\text { Intervention Adherence } \\
\%\end{array}$ & Reporting of adherence to exercise (reported as a percentage) if applicable \\
\hline & AG & Intervention Location & $\begin{array}{l}\text { Location exercise was performed } \\
1=\text { Home; } 2=\text { Clinic } 3=\text { Fitness facility } 4=\mathrm{NR} ; 5=\mathrm{NA}\end{array}$ \\
\hline & $\mathrm{AH}$ & Intervention Volume & Numerical value describing volume \\
\hline & AI & $\begin{array}{l}\text { Intervention Volume } \\
\text { Category }\end{array}$ & $\begin{array}{c}1=\text { Duration of session (mins); } 2=\text { sets } * \text { repetitions; } 3=\text { number of repetitions; } \\
4=\text { number of sets }\end{array}$ \\
\hline & AJ & $\begin{array}{l}\text { Intervention Volume } \\
\text { Comments }\end{array}$ & Any additional information relevant. \\
\hline & $\mathrm{AK}$ & Intervention Intensity & Numerical value describing intensity \\
\hline & $\mathrm{AL}$ & $\begin{array}{l}\text { Intervention Intensity } \\
\text { Category }\end{array}$ & $1=$ Absolute; $2=$ Relative \\
\hline & $\mathrm{AM}$ & Intervention Frequency & $\begin{array}{c}\text { Number of sessions per week. Where there is progression, average value is to be } \\
\text { entered. }\end{array}$ \\
\hline
\end{tabular}

Swinton, Shim, Pavlova, Moss, Maclean, Greig, Parkinson, Morrissey, Alexander, Cooper (2021) 


\begin{tabular}{|c|c|c|c|}
\hline & AN & $\begin{array}{l}\text { Intervention Frequency } \\
\text { Comments }\end{array}$ & Any additional information relevant. \\
\hline & $\mathrm{AO}$ & $\begin{array}{l}\text { Intervention } \\
\text { Progression }\end{array}$ & $\begin{array}{l}\text { Multiple themes to be selected as required } \\
1=\text { No progression; } 2=\text { NR; } 3=\text { Progression volume; } 4=\text { Progression intensity; } \\
5=\text { Progression frequency; } 6=\text { Progression specificity; } 7=\text { Progression capacity; } \\
8=\text { Other }\end{array}$ \\
\hline & $\mathrm{AP}$ & $\begin{array}{l}\text { Intervention } \\
\text { Progression Comments }\end{array}$ & Any additional information relevant. \\
\hline \multirow[b]{2}{*}{ نㅇํㅇ } & AQ & Control Comparator & $1=$ Placebo; $2=$ No treatment \\
\hline & AR & $\begin{array}{l}\text { Control Comparator } \\
\text { Comments }\end{array}$ & Any additional information relevant. \\
\hline \multirow{9}{*}{$\stackrel{\widetilde{\pi}}{\pi}$} & AS & $\begin{array}{l}\text { Intervention Baseline } \\
\text { Mean }\end{array}$ & Baseline mean for exercise therapy \\
\hline & AT & $\begin{array}{l}\text { Intervention Baseline } \\
\text { SD }\end{array}$ & Baseline standard deviation for exercise therapy \\
\hline & $\mathrm{AU}$ & $\begin{array}{l}\text { Intervention } \\
\text { Measurement Mean }\end{array}$ & Mean of outcome for exercise therapy at stated time point \\
\hline & AV & $\begin{array}{l}\text { Intervention } \\
\text { Measurement SD }\end{array}$ & Standard deviation of outcome for exercise therapy at stated time point \\
\hline & $\mathrm{AW}$ & Control Baseline Mean & Baseline mean for control \\
\hline & $\mathrm{AX}$ & Control Baseline SD & Baseline standard deviation for control \\
\hline & AY & $\begin{array}{l}\text { Control Measurement } \\
\text { Mean }\end{array}$ & Mean of outcome for control at stated time point \\
\hline & AZ & $\begin{array}{l}\text { Control Measurement } \\
\text { SD }\end{array}$ & Standard deviation of outcome for control at stated time point \\
\hline & $\mathrm{BA}$ & $\begin{array}{l}\text { Measurement } \\
\text { Comments }\end{array}$ & $\begin{array}{l}\text { State if a different value has been entered for means (e.g. median), a different } \\
\text { value for standard deviations (e.g. standard error, IQR, percentiles, distance from } \\
\text { mean to upper bound). Provide the relevant statistic (width of CI's, width of } \\
\text { percentiles). Also state if data has been extracted by digitization }\end{array}$ \\
\hline
\end{tabular}

\section{* Outcome Specific}

